\title{
Effects of space charge on the mass purification in Penning traps
}

\author{
Alexander Herlert • Christopher Borgmann • \\ Daniel Fink • Christian Holm Christensen • \\ Magdalena Kowalska • Sarah Naimi
}

Published online: 13 April 2011

(C) Springer Science+Business Media B.V. 2011

\begin{abstract}
The influence of space charge on the mass selection of ions stored in a Penning trap was investigated with the ISOLTRAP experiment at CERN/ISOLDE. A mixture of ${ }^{85,87} \mathrm{Rb}^{+}$ions has been used to probe the change of the experimental parameters, e.g. frequencies and amplitudes of the radiofrequency excitations, as a function of the number of ions present in the trap.
\end{abstract}

Keywords Penning trap $\cdot$ Space charge $\cdot$ Radioactive ion beam

\section{Introduction}

Penning traps [1] are used at many radioactive ion-beam facilities for different applications. The possibility to probe the frequency of the cyclotron motion in the confining magnetic field with very high precision allows one to directly measure the mass of stored short-lived ions with an uncertainty below $\delta m / m=1 \times 10^{-8}[2,3]$.

A. Herlert $(\bowtie) \cdot$ M. Kowalska

Physics Department, CERN, 1211 Geneva 23, Switzerland

e-mail: alexander.herlert@yahoo.com

Ch. Borgmann · D. Fink

Max-Planck-Institut für Kernphysik, 69117 Heidelberg, Germany

CH. Holm Christensen

Niels Bohr Institute, University of Copenhagen, 2100 Copenhagen, Denmark

S. Naimi

CSNSM-IN2P3-CNRS, Orsay-Campus, 91405 Paris, France

Present Address:

A. Herlert

School of Physics and Astronomy, University of Manchester,

M13 9PL Manchester, UK 
At the radioactive ion-beam facility ISOLDE at CERN this is realized with the ISOLTRAP mass spectrometer [4]. Another Penning-trap application is the use as buncher and cooler, e.g., for the REX-ISOLDE post-acceleration system at ISOLDE [5]. Furthermore, the WITCH experiment [6] also located at ISOLDE aims at the storage of a large number of ions of short-lived isotopes to probe the recoil energy after beta decay in the trap. In all those cases, the Penning trap is also used to manipulate the ion motion, e.g. for the cooling of the stored ions by collisions with inert buffer gas like helium or neon, and to isolate the ions of interest by use of a mass-selective excitation of the radial motional modes.

The latter is needed since during the production of the radionuclides of interest many other species are created and the resolving power of the separator magnets at ISOLDE is not always sufficient to remove contaminating ions from the beam. Depending on the type of experiment, a small fraction of beam impurities is acceptable. However, for precision mass measurements, e.g. with ISOLTRAP, it is important that only the ions of interest are present, even close lying isomers need to be removed. In case of the WITCH experiment, contaminating short-lived ions are seriously hampering the data acquisition since their recoil ions overlap with those from the ions of interest, thus making it challenging to probe for deviations in the energy distribution of the recoil ions. In addition, since about $10^{5}$ or more ions are stored in each experimental cycle, a higher-abundant contamination might fill up the trap, such that the preparation of the ions of interest will become difficult or even impossible.

A recent example of isobaric contamination is ${ }^{130} \mathrm{Cs}$ in case of mass measurements of ${ }^{130} \mathrm{Cd}$, which is of high interest for the nucleosynthesis $r$ process. Recently, a cooled transfer line has been implemented at the ISOLDE target including a quartz rod to absorb alkali ions [7]. However, this was not enough to sufficiently decrease the cesium contamination and several orders of magnitude more cesium ions were sent to ISOLTRAP together with the short-lived ${ }^{130} \mathrm{Cd}^{+}$ions [8]. Similarly, for WITCH stable ${ }^{35} \mathrm{Cl}^{+}$ions were heavily contaminating the ${ }^{35} \mathrm{Ar}^{+}$ion beam. The resulting large amount of space charge in the buffer-gas filled Penning trap influenced the mass selection performed in the trap, e.g., as reported in [9]. In the following, a first systematic investigation of these space-charge effects is presented.

\section{Experimental setup}

The ISOLTRAP mass spectrometer has been discussed in detail elsewhere [4]. The following is a brief overview: The singly charged ions from ISOLDE are stopped, cooled, and bunched in a linear radiofrequency quadrupole which is filled with helium as buffer gas. For tuning and off-line tests it is also possible to inject ions from a reference ion source which can deliver stable alkali ions like ${ }^{39,41} \mathrm{~K}^{+}$, ${ }^{85,87} \mathrm{Rb}^{+}$, or ${ }^{133} \mathrm{Cs}^{+}$. The amount of injected ions is controlled with a beam gate, an electrostatic deflector either located behind the ISOLDE ion source or the ISOLTRAP ion source. After cooling and bunching the ions are ejected and after passing a pulsed drift tube to lower the energy from $30-60 \mathrm{keV}$ down to about $3 \mathrm{keV}$ they are transferred to the first Penning trap. In this cylindrically shaped trap the ions are further cooled by buffer-gas collisions with helium. The ion motion of the stored ions is manipulated using the buffer-gas centering technique [10] such that 
the ions can be mass separated with a resolving power of the order of $m / \delta m=10^{5}$, i.e. undesired isobars are removed. The purification procedure will be explained in more detail below. After the isobaric cleaning, the ions are ejected and transferred to the precision Penning trap for mass measurement by use of the time-of-flight ioncyclotron-resonance technique [11].

\section{Purification in buffer-gas filled Penning trap}

The method to remove unwanted isobaric contamination is described in [10]. In short, the cleaning procedure has the following sequence: After capturing the ions, their axial motion is cooled by collisions with the buffer-gas atoms ( $\Delta t=150 \mathrm{~ms})$. The magnetron motion of all stored ions is then excited by use of a dipolar $\mathrm{rf}$ excitation $(\Delta t=25 \mathrm{~ms})$ with which the ions are brought on a magnetron radius which is much larger than the radius of the ejection aperture of the trap ( $3 \mathrm{~mm}$ diameter for ISOLTRAP). In the final step, the ions of interest are centered by use of a quadrupolar $\mathrm{rf}$ excitation $(\Delta t=100 \mathrm{~ms})$ which is mass selective, i.e. the excitation frequency is the cyclotron frequency $v_{c}=q B /(2 \pi m)$ with the magnetic field strength $B$, the mass $m$ and the charge $q$ of the ion. This excitation couples the radial motional modes [12] and due to the collisions with the buffer-gas atoms the overall radial motion is damped. In contrast, the unwanted ions are not in resonance, thus they stay on a large magnetron radius and do not pass the ejection aperture during the ejection from the trap.

\section{Results and discussion}

In order to investigate the purification procedure for a larger number of ions, several parameters of the ion preparation and manipulation have been varied. For better comparability, the duration of the rf excitations were kept constant. In particular, the ion response to a quadrupolar rf excitation has been probed for different spacecharge conditions. While in the case of many stored ions an accurate calibration of their number was not yet possible, this number was extrapolated from data for low beam-gate values. In Fig. 1a the number of ions on the Micro-Channel-Plate (MCP) detector after the preparation trap is given as a function of the beam-gate length. It is clearly visible that the ion count does not increase linearly with the beam gate which is most probably due to dead-time effects in the data acquisition. Note that for a large number of ions also a saturation of the MCP channels might occur. In order to check the behavior for larger beam gates, the gain of the MCP was lowered while keeping the threshold of the applied constant fraction discriminator constant. This resulted in correspondingly decreased ion counts (Fig. 1b). The slopes for different gain values are comparable and suggest that the number of ions is indeed increased when opening the beam gate for longer times. For an estimate on the number of stored ions, only very low beam gates were taken which show a linear trend in the ion count as shown in Fig. 1c for ${ }^{85} \mathrm{Rb}^{+}$and ${ }^{87} \mathrm{Rb}^{+}$ions. Note that for this measurement a different setting of the ion source was chosen as compared with (a) and (b), i.e. a smaller number of ions per $\mu$ s. These settings were kept for the following measurements. The ratio of the fitted slopes, $0.398(7)$, is in good agreement 

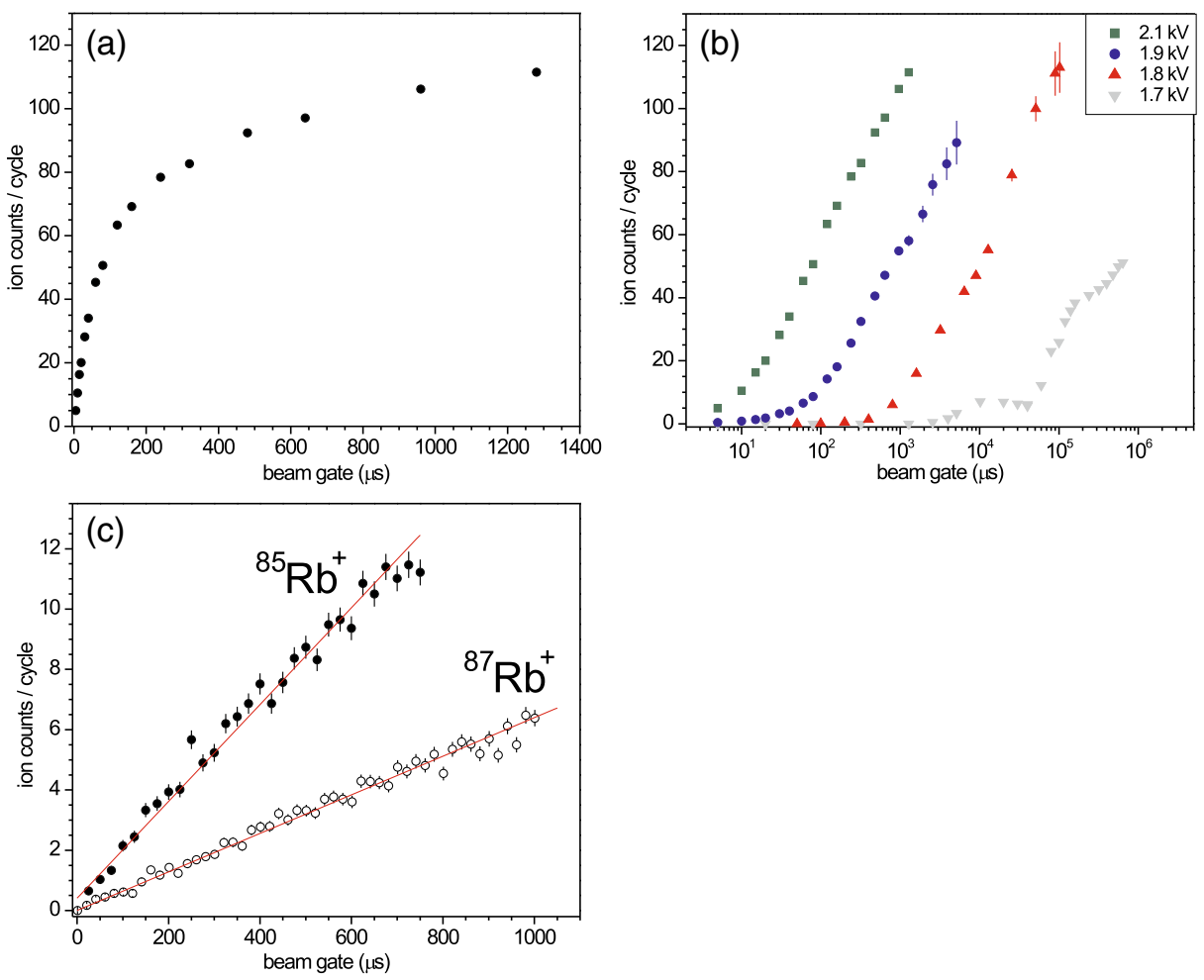

Fig. 1 a Number of detected ${ }^{85} \mathrm{Rb}^{+}$ions per experimental cycle as a function of the beam-gate length. The ions have been counted with the MCP detector mounted behind the purification Penning trap $\left(U_{M C P}=2.1 \mathrm{kV}\right)$. b Number of ${ }^{85} \mathrm{Rb}^{+}$ions per experimental cycle as a function of the beamgate length for different voltages applied to the MCP detector. The beam gate is given with a logarithmic scale. c Number of ions counted with the MCP detector after the purification trap as a function of the beam-gate length for ${ }^{85} \mathrm{Rb}^{+}$(filled circles) and ${ }^{87} \mathrm{Rb}^{+}$(empty circles). The solid lines are linear fits to the data with the slopes $0.01605(23)$ ions $/ \mu$ s and $0.00639(6)$ ions $/ \mu$ s. The source settings are different from the data in $\mathbf{a}$ and $\mathbf{b}$ (see text)

with the value 0.389 which is expected from the ratio of the natural abundance of the two rubidium isotopes. Under the same ion-source conditions a much higher ${ }^{133} \mathrm{Cs}^{+}$ ion yield was observed with a slope of $0.612(5)$ ions $/ \mu$ s. In the following, only the beam-gate values are given. An estimate of the number of stored ions can be deduced taking the calibration from Fig. 1 and assuming an efficiency of the MCP detector of about $30 \%$. However, an extrapolation to higher beam gates can lead to large deviations between the expected and actual ion number.

A first attempt to probe the space-charge effect was performed with ${ }^{85} \mathrm{Rb}^{+}$ stored in the purification trap together with the lower-abundant isotope ${ }^{87} \mathrm{Rb}^{+}$, both delivered from the ion source. Cyclotron resonance curves were recorded for different beam-gate lengths (Fig. 2a). Obviously, both a broadening and a shift of the center of the resonance are observed. The broadening might be due to the saturation of the MCP detector for high ion numbers. When the gain of the MCP is reduced (Fig. 2b), the width of the resonance increases slightly if the beam-gate length is increased while the frequency shift shows a similar trend (Fig. 2c and d). 
(a)

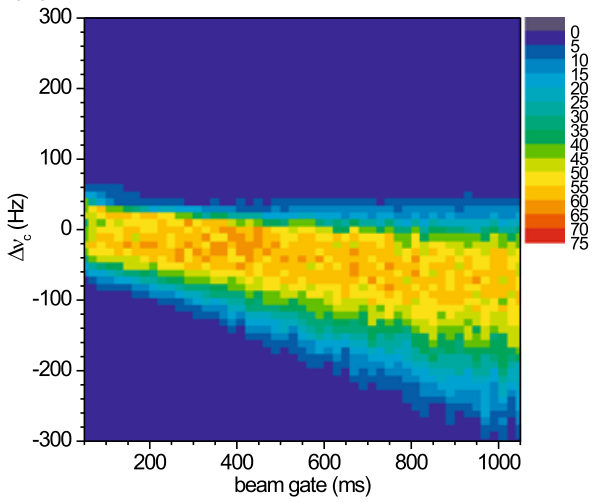

(c)

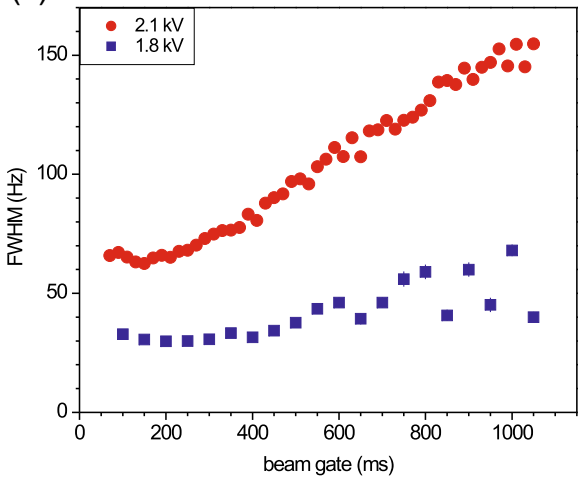

(b)

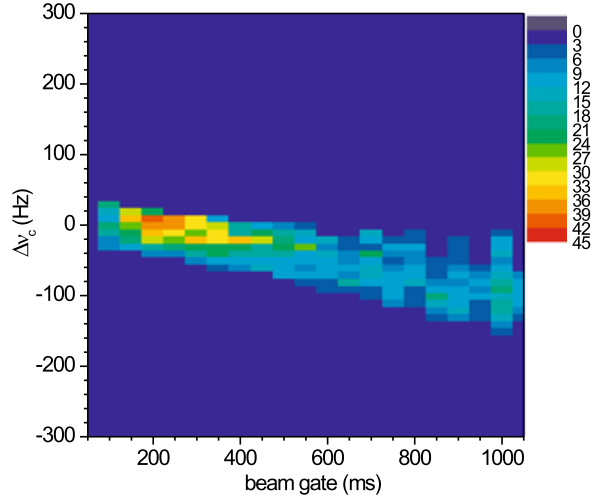

(d)

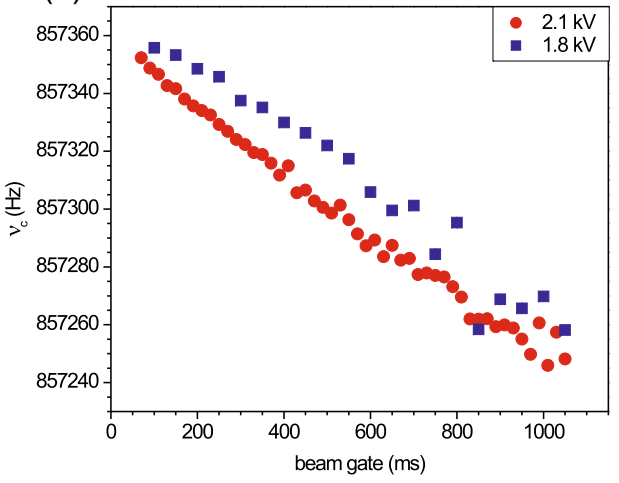

Fig. 2 Top: Number of ${ }^{85} \mathrm{Rb}^{+}$ions measured with the MCP detector behind the purification Penning trap as a function of the beam-gate length and the quadrupolar if frequency $v_{r f}$ relative to the expected value of the expected cyclotron frequency $v_{c}\left(\Delta v_{c}=v_{r f}-v_{c}\right)$. The voltage of the MCP was set to $\mathbf{a} U_{M C P}=2.1 \mathrm{kV}$ and $\mathbf{b} U_{M C P}=1.8 \mathrm{kV}$. In both cases the amplitude of the dipolar rf excitation of the magnetron motion was $A_{m a g}=0.9 \mathrm{~V}_{P P}$ and of the quadrupolar rf excitation $A_{c y c l}=1.1 \mathrm{~V}_{P P}$. Bottom: c FWHM of the cyclotron-resonance curves (from a and $\mathbf{b}$ ) as a function of the beamgate length for $U_{M C P}=1.8 \mathrm{kV}$ (squares) and $U_{M C P}=2.1 \mathrm{kV}$ (circles). d Cyclotron frequencies $v_{c}$ from data in $\mathbf{a}$ and $\mathbf{b}$ as a function of the beam-gate length for $U_{M C P}=1.8 \mathrm{kV}$ (squares) and $U_{M C P}=2.1 \mathrm{kV}($ circles $)$

In a previous investigation [9], ${ }^{39} \mathrm{~K}^{+}$and ${ }^{41} \mathrm{~K}^{+}$ions were simultaneously stored in the purification trap (natural abundance of ${ }^{39} \mathrm{~K}$ is $93.3 \%$ and of ${ }^{41} \mathrm{~K} 6.7 \%$ ). When the total number of ions was varied by changing the beam gate of the ion source, the cyclotron resonance of ${ }^{41} \mathrm{~K}^{+}$showed a splitting due to the presence of ${ }^{39} \mathrm{~K}^{+}$. This behavior was reproduced in the present work for a mixture of ${ }^{85} \mathrm{Rb}^{+}$and ${ }^{87} \mathrm{Rb}^{+}$under similar conditions and with natural abundances of 72.2 and $27.8 \%$, respectively. Figure 3 shows the number of observed ${ }^{87} \mathrm{Rb}^{+}$ions as a function of the beam-gate length and the applied quadrupolar rf excitation. While for small beam-gate lengths the cyclotron resonance is observed at the expected frequency $v_{c}$, a splitting occurs into a broader part at lower frequencies and a narrow peak at higher frequencies. Increasing the number of ions increases the gap between the two regions. This is in contrast to the case of ${ }^{85} \mathrm{Rb}^{+}$where no splitting was observed. In order to make a 
Fig. 3 Number of measured beam-gate length and the frequency of the applied quadrupolar rf excitation $v_{r f}$, the latter relative to the expected cyclotron frequency measured for only a few ions stored in the purification trap. The amplitude of the dipolar $\mathrm{rf}$ excitation was $A_{m a g}=1.2 \mathrm{~V}_{P P}$ and of the quadrupolar $\mathrm{rf}$ excitation $A_{c y c l}=1.8 \mathrm{~V}_{P P}$
${ }^{87} \mathrm{Rb}^{+}$ions as a function of the $v_{c}\left(\Delta v_{c}=v_{r f}-v_{c}\right)$ which was

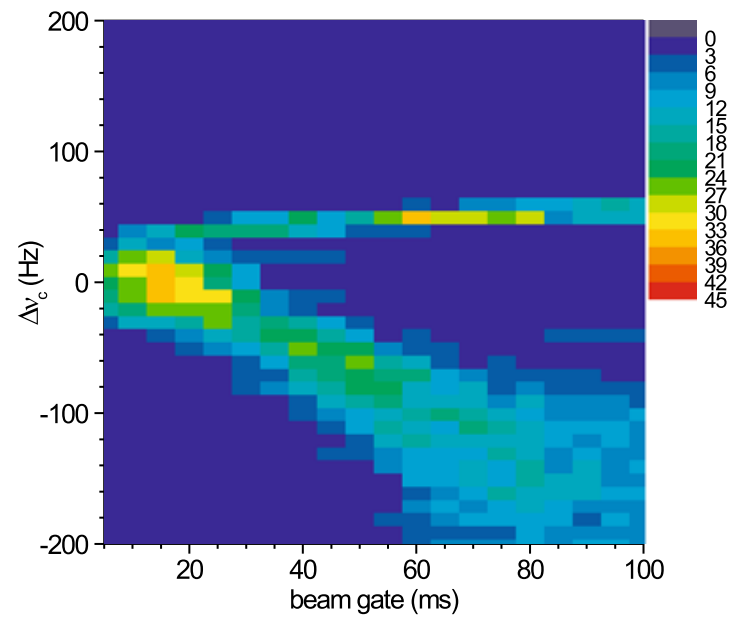

(b)
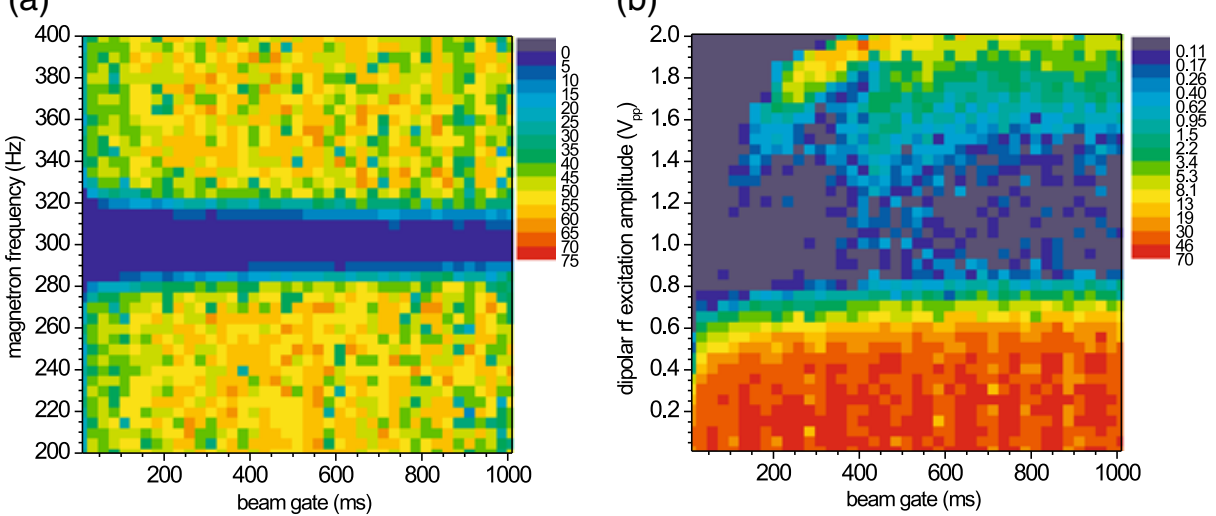

Fig. 4 a Number of ${ }^{87} \mathrm{Rb}^{+}$ions as a function of the beam-gate length and the frequency of the applied dipolar rf excitation of the magnetron motion. The amplitude of the dipolar rf excitation was $A_{\text {mag }}=0.8 \mathrm{~V}_{P P}$. b Number of ${ }^{87} \mathrm{Rb}^{+}$ions as a function of the beam-gate length and the amplitude of the dipolar rf excitation. The frequency of the excitation was fixed at $v_{r f}=300 \mathrm{~Hz}$. The scale of the ion count is logarithmic to enhance features at low ion yields

more systematic approach, several parameters of the cleaning procedure have been varied for different space-charge conditions.

The first parameter of the cleaning procedure that was probed was the magnetron frequency. In Fig. 4a the frequency of a dipolar rf excitation has been varied around the expected magnetron frequency for different beam-gate lengths. The amplitude of the excitation was chosen in such a way to increase the magnetron radius well beyond the exit diaphragm towards the ion detector. No significant shift of the magnetron frequency due to space charge is observed. Keeping the frequency of the dipolar rf excitation fixed and varying the amplitude gives a result as shown in Fig. 4b. While for low beam gates the ions are not observed above a certain threshold value of the amplitude, a few ions reappear at higher space-charge values (note the logarithmic 
(a)

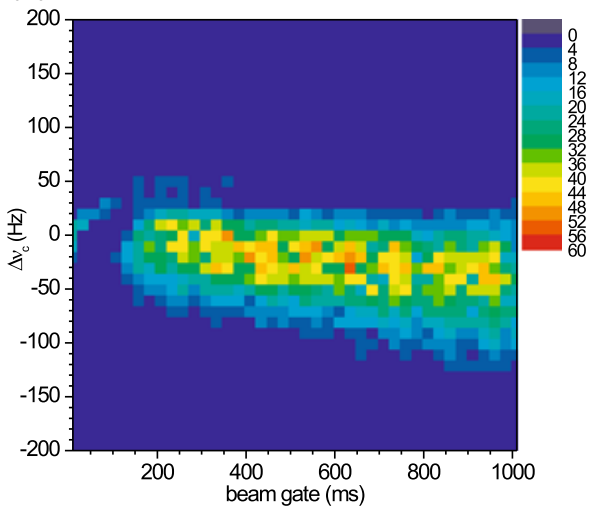

(c)

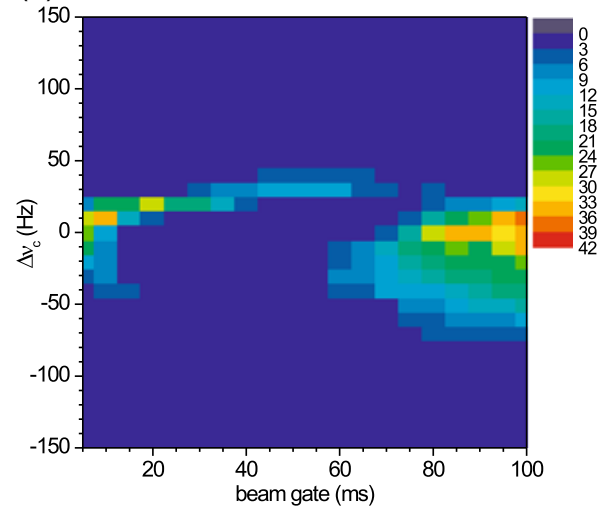

(b)

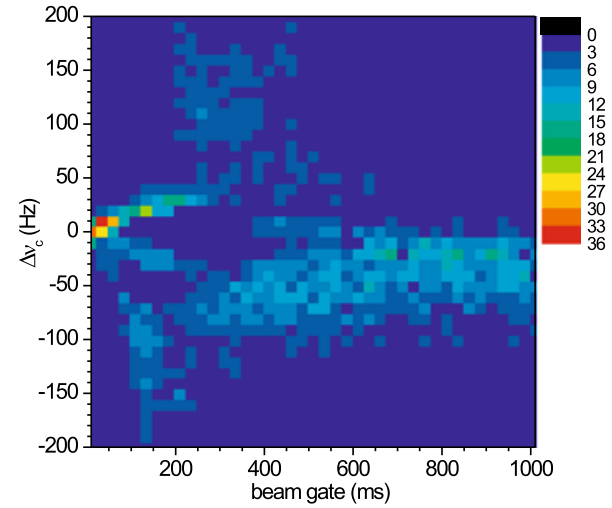

Fig. 5 a Number of ${ }^{87} \mathrm{Rb}^{+}$ions as a function of the beam-gate length and the frequency of the applied quadrupolar rf excitation relative to the expected cyclotron frequency at low beam gate. The amplitude of the dipolar rf excitation was $A_{m a g}=0.9 \mathrm{~V}_{P P}$ and of the quadrupolar rf excitation $A_{c y c l}=1.0 \mathrm{~V}_{P P}$. b Same as a with a higher amplitude $A_{m a g}=1.2 \mathrm{~V}_{P P}$ for the dipolar rf excitation of the magnetron motion. $\mathbf{c}$ Data remeasured for parameters as given in a for smaller beam gates

scale of the false-color plot) for higher amplitudes. This behavior might be due to the Coulomb interaction of the ions while circulating on the magnetron trajectory [13].

The cyclotron frequency was also measured for the less abundant ${ }^{87} \mathrm{Rb}^{+}$having ${ }^{85} \mathrm{Rb}^{+}$stored simultaneously in the purification trap. Varying the quadrupolar $\mathrm{rf}$ excitation frequency for different beam-gate lengths, i.e. for different numbers of stored ions, gives the result as shown in Fig. 5a. The general trend is the same as observed for ${ }^{85} \mathrm{Rb}^{+}$, i.e. a linear decrease of the cyclotron frequency. However, at low beam gates, between a few tens of ms and $200 \mathrm{~ms}$, almost no ions are detected. If the amplitude of the dipolar rf excitation $A_{m a g}$ is increased to $1.2 \mathrm{~V}_{P P}$ the behavior changes completely as shown Fig. 5b. Only at low beam gates a regular cyclotron resonance is observed. Otherwise, the frequency scans show a 'deformed' resonance shape. Figure 5c gives a close up of the scan region for parameters as applied in (a). Obviously, under all these conditions, the mass selection does not work properly. 
Fig. 6 Number of ${ }^{87} \mathrm{Rb}^{+}$ions as a function of the beam-gate length and the amplitude of the applied quadrupolar rf excitation with $v_{r f}$ kept at the expected cyclotron frequency. The amplitude of the dipolar rf excitation of the magnetron motion was $A_{m a g}=1.2 \mathrm{~V}_{P P}$

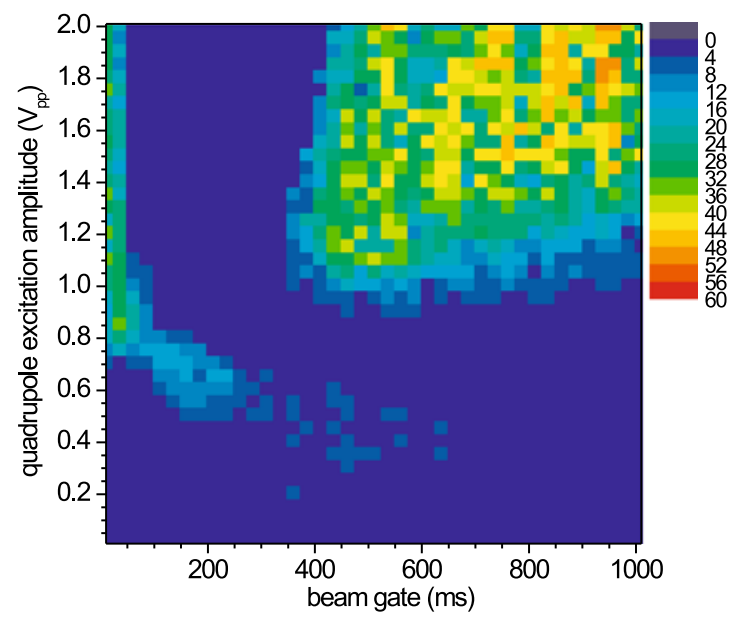

(a)

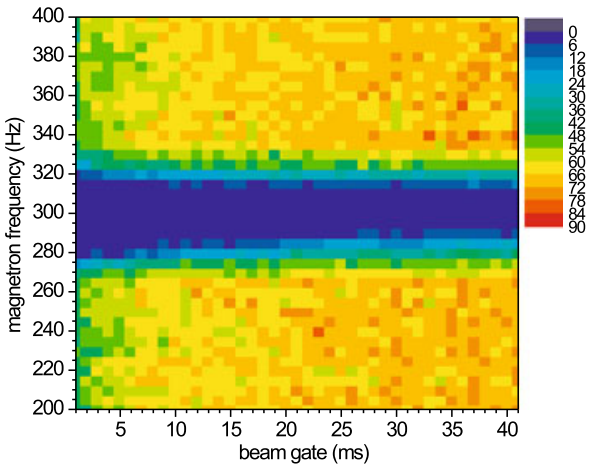

(b)

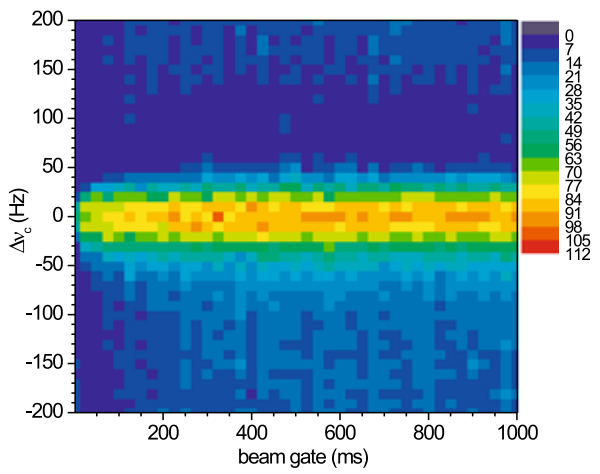

Fig. 7 a Number of ${ }^{133} \mathrm{Cs}^{+}$ions as a function of the beam-gate length and the frequency of the dipolar rf excitation of the magnetron motion. The amplitude of the dipolar rf excitation was fixed at $A_{\text {mag }}=0.9 \mathrm{~V}_{P P}$. b Number of ${ }^{133} \mathrm{Cs}^{+}$ions as a function of the beam-gate length and the frequency of the quadrupolar rf excitation relative to the expected cyclotron frequency. The amplitude of the quadrupolar rf excitation was $A_{c y c l}=0.5 \mathrm{~V}_{P P}$

Keeping the frequency of the quadrupolar $\mathrm{rf}$ excitation fixed at the expected frequency $v_{c}$ and varying the amplitudes for different beam gates gives the result shown in Fig. 6. For low beam gates, i.e. less than $100 \mathrm{~ms}$ under the present ion source conditions, a threshold value of $A_{c y c l}$ is observed which is needed for a full conversion from the magnetron motion to the cyclotron motion which itself is cooled by the buffer-gas collisions. Increasing the amplitude further only results in a plateau for the ion yield. If the beam gate is increased this expected and well-understood behavior changes completely. First, for only a small amplitude range around $A_{c y c l}=0.6 \mathrm{~V}_{P P}$ ions are observed. Second, almost all ions disappear for beam gates around $300 \mathrm{~ms}$. Finally, the plateau reappears if the beam-gate length is further increased.

As a final test in this study, a single isotope ion bunch was examined. ${ }^{133} \mathrm{Cs}^{+}$ ions from the alkali ion source were injected into the purification trap and also the 
behavior of the ion manipulation with rf excitations checked for different numbers of stored ions. The magnetron frequency was measured as a function of the applied beam-gate length. No significant shift is observed as shown in Fig. 7a. Similar to the measurement for the rubidium isotopes, the cyclotron frequency was determined by use of a scan of the quadrupolar rf frequency and the number of ions was increased by increasing the beam-gate length. In contrast to the results obtained for rubidium under similar conditions, no frequency shift or splitting is observed (Fig. 7b). Note that the yield of ${ }^{133} \mathrm{Cs}^{+}$ions from the ISOLTRAP ion source is much larger than for the rubidium ions. Therefore the total number of stored ions is comparable to the one in the measurements for the rubidium isotopes mentioned above. Since only one isotope is present in the trap, there is no interaction of two different species orbiting on different magnetron radii. This Coulomb interaction was used in [13] to simulate the splitting of the cyclotron resonance curve as reported in [9] and measured for ${ }^{87} \mathrm{Rb}^{+}$ions in this work.

\section{Conclusion and outlook}

A shift of the cyclotron frequency of ${ }^{85,87} \mathrm{Rb}^{+}$ions to lower frequencies is observed in the purification Penning trap of ISOLTRAP when applying the standard massselection procedure under space-charge conditions. For the magnetron frequency no shift was observed. In the case of ${ }^{133} \mathrm{Cs}^{+}$ions, no shifts or splitting of the cyclotron resonances were observed, probably due to the missing mixture of ion species in the trap, i.e. no Coulomb interaction with ions staying on a large magnetron orbit. At the REX-ISOLDE Penning trap system REXTRAP, space-charge effects were also observed recently which are similar to the present results [14]. Further studies are needed to understand this behavior and to improve the mass purification technique when more than several hundred ions are present in the trap. Especially the centering for very low abundant ion species in the presence of extensive isobaric contamination has to be further investigated before measurements on rare isotopes like ${ }^{130} \mathrm{Cd}$ will become feasible.

In addition to cleaning with the buffer-gas centering method, alternative techniques have to be applied: The purification of radioactive ion beams in the target/ionsource unit is essential and may help to reduce contamination of exotic beams. Several techniques have been tested and are promising, e.g. the application of a quartz transfer line. Nevertheless, further development is needed like new laser-ionsource schemes and new target materials. At ISOLTRAP, a multi-reflectron TOF system has been installed recently [15]. With this device, it is planned to reduce the amount of isobaric contamination before injection of ions into the purification trap. This will possibly reduce the space charge to operational conditions or even remove it completely. It was also tried to eject unwanted ions from the purification trap using a dipolar rf excitation [9]. However, further tests are needed to examine the limitations and the efficiency.

A recent approach [16] to perform ab-initio calculations of the ion motion of more than 1000 simultaneously stored ions taking into account buffer-gas collisions and Coulomb interactions might shed some light on the results obtained in the present work. In particular, the dependence of the cyclotron cooling on the abundance ratio and absolute numbers of the two (or more) stored ion species has to be further 
investigated. Realistic on-line beam conditions have to be studied to determine the present limitations and to improve the purification procedures.

Acknowledgements The authors acknowledge the support from the Max-Planck Society. Ch.H.Ch. thanks CERN for support during the Summer Student program 2009.

\section{References}

1. Brown, L.S., Gabrielse, G.: Rev. Mod. Phys. 58, 233 (1986)

2. Blaum, K.: Phys. Rep. 425, 1 (2006)

3. Schweikhard, L., Bollen, G. (eds.): Int. J. Mass Spectrom. 251(2-3), 85-312 (2006)

4. Mukherjee, M., et al.: Eur. Phys. J. A 35, 1 (2008)

5. Voulot, D., et al.: Nucl. Instrum. Methods B 266, 4103 (2008)

6. Beck, M., et al.: Nucl. Instrum. Methods A 503, 567 (2003)

7. Bouquerel, E., et al.: Eur. Phys. J. Special Topics 150, 277 (2007)

8. Breitenfeldt, M., et al.: Phys. Rev. C 81, 034313 (2010)

9. Schweikhard, L., Blaum, K., Herlert, A., Marx, G.: Eur. J. Mass Spectrom. 11, 457 (2005)

10. Savard, G., et al.: Phys. Lett. A 158, 247 (1991)

11. Gräff, G., et al.: Z. Phys. A 297, 35 (1980)

12. Bollen, G., Moore, R.B., Savard, G., Stolzenberg, H.: J. Appl. Phys. 68, 4355 (1990)

13. Sturm, S., et al.: AIP Proceedings 1114, 185 (2009)

14. Gustafsson, A., Herlert, A., Wenander, F.: Nucl. Instrum. Methods A 626-627, 8 (2011)

15. Wolf, R.N., et al.: Hyperfine Interact. (this issue, submitted). doi:10.1007/s10751-011-0306-8

16. Van Gorp, S., et al.: Nucl. Instrum. Methods A 638, 192 (2011) 